Lucas Gabriel Maltoni Romano ${ }^{1}$

GiUliano BeDOSCHI ${ }^{2}$

ANDERSON SANCHES MELO ${ }^{3}$

Felipe Oliveira de Albuquerque ${ }^{3}$

ana Carolina Japur de Sá Rosa e Silva ${ }^{4}$

RUI Alberto FerRIAN ${ }^{5}$

Paula ANDREa NaVARRO 4

Artigo original

\title{
Anormalidades metabólicas em mulheres com síndrome dos ovários policísticos: obesas e não obesas
}

\author{
Metabolic abnormalities in polycystic ovary syndrome women: \\ obese and non obese
}

Palavras-chave

Síndrome do ovário policístico

Obesidade

Resistência à insulina

Dislipidemias

Keywords

Polycystic ovary syndrome

Obesity

Insulin resistance

Dyslipidemias

\section{Resumo}

OBJETIVO: Comparar as características metabólicas de mulheres jovens do sudeste brasileiro, obesas e não obesas com síndrome dos ovários policísticos (SOP). MÉTODOS: Estudo transversal que incluiu 218 mulheres de idade reprodutiva com diagnóstico de SOP - 90 mulheres não obesas (IMC entre 18,5 e 29,9kg/m²) e 128 pacientes obesas (IMC $\left.\geq 30 \mathrm{~kg} / \mathrm{m}^{2}\right)$, selecionadas no momento do diagnóstico. Foram comparadas as frequências de resistência insulínica (RI), intolerância à glicose (IG), síndrome metabólica (MetS) e diabetes mellitus tipo 2 $\left(D M_{2}\right)$ e os valores médios de colesterol total (CT), triglicérides (TG), lipoproteínas de alta (LDL) e baixa densidade $(H D L)$, entre as pacientes obesas e não obesas com SOP. Foram comparadas também as características clínicas e hormonais (hormônio folículo estimulante, luteinizante, prolactina, hormônio tireoestimulante, testosterona total, sulfato de dehidroepiandrostenediona e 17-hidroxiprogesterona) nos dois grupos. A análise estatística foi realizada com o auxílio do software SAS 9.0. Para análise das variáveis com distribuição normal, utilizou-se o teste t de Student não pareado; na ausência desta característica, o teste utilizado foi Mann-Whitney bicaudal. Para as variáveis qualitativas utilizou-se o teste Exato de Fisher. Em todas as análises, foi considerado o nível de significância de 5\% ( $<<0,05)$. RESULTADOS: As frequências de RI, IG e MetS foram significativamente mais elevadas em pacientes com SOP obesas do que não obesas $(66,7,29,9$ e $63 \%$ versus $24,7,12,2$ e 16.4\%, respectivamente). As pacientes obesas apresentaram maiores níveis de CT e TG $(189,8 \pm 35.8 \mathrm{mg} / \mathrm{dL}$ e $145.4 \pm 71.1 \mathrm{mg} / \mathrm{dL}$, respectivamente) do que as não obesas $(172,1 \pm 38.4 \mathrm{mg} / \mathrm{dL}$ e 99,3 $\pm 54 \mathrm{mg} / \mathrm{dL}$, respectivamente). Ambos os grupos apresentaram níveis médios de HDL abaixo de $50 \mathrm{mg} / \mathrm{dL}$. CONCLUSÕES: Mulheres obesas jovens com SOP apresentam maior frequência de RI, IG e SM do que as não obesas. Todavia, a ocorrência dos distúrbios metabólicos é elevada também na pacientes não obesas, sugerindo que a presença da síndrome favoreça o desenvolvimento de comorbidades metabólicas, com potenciais repercussões a médio e longo prazos.

\section{Abstract}

PURPOSE: To compare the metabolic characteristics of obese and non-obese young women with polycystic ovary syndrome (POS) from the Brazilian Southeast. METHODS: This was a cross-sectional study conducted on 218 women of reproductive age with a diagnosis of POS - 90 non-obese women (BMI between 18.5 and $\left.29.9 \mathrm{~kg} / \mathrm{m}^{2}\right)$, and 128 obese patients $\left(B M I \geq 30 \mathrm{~kg} / \mathrm{m}^{2}\right)$ selected at the time of diagnosis. The frequency of insulin resistance (IR), glucose intolerance $(G I)$, metabolic syndrome (MetS) and type 2 diabetes mellitus $\left(D M_{2}\right)$ and mean values of total cholesterol $(T C)$, triglycerides (TG), high-density (HDL) and low-density lipoproteins (LDL), were compared between obese and nonobese patients with POS. The two groups were also compared in terms of clinical and hormonal characteristics (follicle stimulating hormone, prolactin, thyroid stimulating hormone, total testosterone, dihydroepiandrostenedione sulfate, and 17-hydroxyprogesterone). Statistical analysis was performed using the SAS 9.0 software. Quantitative variables were compared by the Student's ttest (data with normal distribution) or by the Mann-Whitney test (non-parametric distribution). Qualitative variables were compared by the Fisher test. The level of significance was set at $5 \%(p<0.05)$ in all analyses. RESULTS: The frequency of IR, GI and MetS was significantly higher in obese than non-obese patients
Correspondênnia:

Paula Andrea Navarro Setor de Reprodução Humana, Departamento de Ginecologia e Obstetrícia da Faculdade de Medicina de Ribeir̃õo Preto da Universidade de São Paulo Av. Bandeirantes, 3.900 - Monte Alegre (EP: 14049-900 - Ribeir̃̃o Preto (SP), Brasil Tel.: (16) 3602-2821 - Fax: (16) 3602-2810

Recebido 20/06/2011

Aceito com modificacōoses $30 / 06 / 2011$
Setor de Reprodução Humana, Departamento de Ginecologia e Obstetrícia da Faculdade de Medicina de Ribeirão Preto da Universidade de São Paulo - FMRP-USP - Ribeirão Preto (SP), Brasil.

' Médico Residente do Departamento de Neurociências e Ciências do Comportamento do Hospital das Clínicas de Ribeirão Preto da Faculdade de Medicina de Ribeirão Preto da Universidade de São Paulo - FMRP-USP - Ribeirão Preto (SP), Brasil.

${ }^{2}$ Médico Residente do Departamento de Ginecologia e Obstetrícia do Hospital das Clínicas de Ribeirão Preto da Faculdade de Medicina de Ribeirão Preto da Universidade de São Paulo - FMRP-USP - Ribeirão Preto (SP), Brasil.

${ }_{3}$ Médico Assistente do Departamento de Ginecologia e Obstetrícia do Hospital das Clinicas de Ribeirão Preto da Faculdade de Medicina de Ribeirão Preto da Universidade de São Paulo - FMRP-USP - Ribeirão Preto (SP), Brasil.

${ }_{4}^{4}$ Professora Doutora do Departamento de Ginecologia e Obstetrícia, Setor de Reprodução Humana, da Faculdade de Medicina de Ribeirão Preto da Universidade de São Paulo - FMRP-USP -Ribeirão Preto (SP), Brasil.

${ }_{5}^{5}$ Professor Titular do Departamento de Ginecologia e Obstetrícia da Faculdade de Medicina de Ribeirão Preto da Universidade de São Paulo - FMRP- USP - Ribeirão Preto (SP), Brasil.

Fonte de Auxílio: Fundação de Amparo à Pesquisa do Estado de São Paulo - FAPESP - (2008/57640-3) e Instituto Nacional de Ciências e Tecnologia - INCT/CNPq - Hormônios e Saúde da Mulher. 
with POS (66.7, 29.9, and 63\% versus 24.7, 12.2, and 16.4\%, respectively). Obese patients had higher TC and TG levels (189.8 $\pm 35.8 \mathrm{mg} / \mathrm{dL}$ and $145.4 \pm 71.1 \mathrm{mg} / \mathrm{dL}$, respectively) than non-obese patients $(172.1 \pm 38.4 \mathrm{mg} / \mathrm{dL}$ and $99.3 \pm 54 \mathrm{mg} / \mathrm{dL}$, respectively). Both groups had mean HDL levels below 50 mg/dL. CONCLUSIONS: Young obese women with POS have a higher frequency of IR, GI and MS than non-obese. However, the occurrence of metabolic disorders is elevated also in the non-obese patients, suggesting that the presence of the syndrome may favor the development of metabolic comorbidities with potential medium- and long-term repercussions.

\section{Introdução}

A síndrome dos ovários policísticos (SOP) é uma desordem endócrina heterogênea e complexa com prevalência estimada entre 4 e $13 \%$ das mulheres em idade reprodutiva ${ }^{1}$. Trata-se da principal causa de hiperandrogenismo e oligo-anovulação, sendo comum sua associação com distúrbios metabólicos ${ }^{2}$.

As alterações clínicas e metabólicas da SOP estão relacionadas principalmente ao hiperandrogenismo ${ }^{3} \mathrm{e}$ à resistência insulínica $(\mathrm{RI})^{4,5}$. Como menos de $20 \%$ das pacientes magras com SOP apresentam alterações metabólicas relacionadas à $\mathrm{RI}^{6,7}$, é possível que haja uma superestimativa da relação entre a RI e as repercussões metabólicas desta síndrome neste grupo de mulheres. Nestas situações, o hiperandrogenismo pode desempenhar papel fundamental no desenvolvimento de comorbidades metabólicas ${ }^{8}$.

A RI e a obesidade centrípeta são os principais fatores determinantes da síndrome metabólica (MetS), um fator de risco importante para o desenvolvimento da doença cardiovascular aterosclerótica e do diabetes mellitus tipo $2\left(\mathrm{DM}_{2}\right)^{9}$. Como a prevalência de RI e da MetS está aumentada em mulheres com SOP, é possível que estas pacientes apresentem maior risco para eventos cardiovasculares ${ }^{10} \mathrm{e}$ metabólicos. Este risco pode ser ainda maior na presença da obesidade ${ }^{11}$.

A obesidade ocorre em 30 a $70 \%$ das pacientes com $\mathrm{SOP}^{12}$, podendo ser considerada como variante do processo que predispõe à síndrome. Evidências sugerem que alterações intrauterinas possam determinar uma cascata de efeitos metabólicos ao longo da vida da mulher (restrição de crescimento intrauterino, hiperinsulinemia, obesidade, hiperandrogenismo e, finalmente, a SOP). Assim, a RI pode estar associada ao desenvolvimento de comorbidades metabólicas ${ }^{1,5} \mathrm{em}$ mulheres obesas com SOP. Por outro lado, pacientes magras podem apresentar variantes genéticas relacionadas ao hiperandrogenismo, mas não associadas a alguns critérios da MetS ${ }^{13}$. Estas características sugerem que o risco metabólico de mulheres com SOP possa ser diferente de acordo com o índice de massa corpórea (IMC), apesar da etiologia multifatorial destas alterações ${ }^{1,14}$.

Como a SOP e seus distúrbios metabólicos apresentam prevalência variável de acordo com a etnia e outras especificidades da população estudada ${ }^{3}$, é fundamental caracterizar a prevalência destes distúrbios e avaliar se existem riscos específicos identificáveis para determinado grupo populacional, o que poderá contribuir para a definição de medidas precoces de prevenção e tratamento. Assim, este estudo teve como objetivo geral comparar as características metabólicas de mulheres jovens obesas e não obesas do sudeste brasileiro com SOP.

\section{Métodos}

\section{Pacientes}

Foi realizado um estudo transversal avaliando 218 mulheres de idade reprodutiva com diagnóstico de SOP realizado segundo os critérios do Consenso de Rotterdam ASRM/ESHRE ${ }^{15}$. Estas pacientes foram divididas em dois grupos de acordo com o IMC: (1) grupo não obesa, constituído por 90 mulheres com IMC entre 18,5 e $29,9 \mathrm{~kg} /$ $\mathrm{m}^{2} \mathrm{e}$, (2) grupo obesa, com 128 pacientes com IMC $\geq 30$. Como objetivos primários foram comparadas as frequências de resistência insulínica (RI), intolerância à glicose (IG), síndrome metabólica (SM) e diabetes mellitus tipo $2\left(\mathrm{DM}_{2}\right)$ entre mulheres com SOP obesas e não obesas. Também foram comparados os valores médios de colesterol total (CT), triglicérides (TG), lipoproteínas de alta (LDL) e baixa densidade (HDL), entre os grupos. Foram comparadas também as características clínicas e hormonais (hormônio folículo estimulante, luteinizante, prolactina, hormônio tireoestimulante, testosterona total, sulfato de dehidroepiandrostenediona e 17-hidroxiprogesterona) dos dois grupos.

As mulheres com SOP foram selecionadas do ambulatório de endocrinologia ginecológica do Hospital das Clínicas da Faculdade de Medicina de Ribeirão Preto da Universidade de São Paulo (HC-FMRP-USP) durante o período de 2004 a 2008. A inclusão destas mulheres foi realizada imediatamente após o diagnóstico da SOP.

Como critérios de exclusão foram considerados a gestação/amamentação; uso atual ou nos últimos três meses de medicações antiandrogênicas ou anti-inflamatórias; uso atual ou nos últimos três meses de anticoncepcional oral, vaginal ou transdérmico; uso de método contraceptivo hormonal injetável, implante ou dispositivo intrauterino no período de seis meses antes do diagnóstico de SOP; tabagismo (consumo de qualquer quantidade de cigarro ou outros produtos que contenham nicotina); endocrinopatias associadas à anovulação e hiperandrogenismo (hiperprolactinemia, hiperplasia congênita da 
suprarrenal formas clássica e não-clássica, tireoidopatias, tumores produtores de androgênio e síndrome/doença de Cushing); presença de doenças autoimunes; dislipidemia familiar; doença cardiovascular estabelecida e puerpério menor ou igual a 12 semanas. Todas as participantes deste estudo assinaram termo de consentimento livre e esclarecido e o estudo foi aprovado pelo Comitê de Ética do HC-FMRP-USP.

Considerando-se os critérios diagnósticos de SOP de acordo com o Consenso de Rotterdam, no total de pacientes avaliadas, observou-se que a anovulação ocorreu em 93,1\% das pacientes, sendo o critério mais frequente no diagnóstico de SOP. Oitenta e oito por cento das pacientes apresentaram critérios ecográficos de ovário policístico e $55,6 \%$, hiperandrogenismo clínico. O hiperandrogenismo laboratorial foi a variável com menor frequência, estando presente em $39,7 \%$ do total das pacientes.

Em relação à avaliação clínica, observou-se que a idade média das pacientes obesas no momento do diagnóstico foi significativamente maior do que a das não obesas $(27,8 \pm 5,1$ anos e 23,3 $\pm 4,55$ anos, respectivamente; $p<0,001)$. Não se observou diferença significativa no volume ovariano médio entre os grupos, cujos valores foram maiores do que o limite superior da normalidade do volume ovariano de mulheres na menacme $\left(9 \mathrm{~cm}^{3}\right)$ (Tabela 1$)$.

\section{Protocolos de avaliação clínica e laboratorial}

Os dados clínicos e laboratoriais foram obtidos dos registros de inclusão no ambulatório de endocrinologia ginecológica do HC-FMRP-USP, imediatamente após o diagnóstico da anovulação crônica. A avaliação laboratorial e ultrassonográfica foram realizadas entre o terceiro e o quinto dia do ciclo menstrual, após período de jejum de 12 horas. Na presença de amenorréia, os exames foram realizados em programação aleatória (desde que a ultrassonografia não mostrasse imagem de corpo lúteo ou folículo $\geq 10 \mathrm{~mm}$ ).

Em relação à anamnese e ao exame físico, foram consideradas as seguintes variáveis: idade no momento

Tabela 1. Caracterização clínica das pacientes obesas e não obesas com síndrome dos ovários policísticos (SOP)

\begin{tabular}{l|ccc}
\hline Variável & SOP obesas $\mathrm{n}=128$ & SOP não obesas $\mathrm{n}=90$ & Valor $\mathrm{p}$ \\
\hline Idade (anos) & $27,8 \pm 5,1$ & $23,3 \pm 4,6$ & $<0,001$ \\
\hline Peso $(\mathrm{kg})$ & $97,9 \pm 16,9$ & $61,4 \pm 10,4$ & $<0,001$ \\
\hline Estatura $(\mathrm{m})$ & $1,6 \pm 0,1$ & $1,64 \pm 0,1$ & NS \\
IMC (m/ $\left./ \mathrm{kg}^{2}\right)$ & $38,2 \pm 6,0$ & $23,9 \pm 3,6$ & $<0,001$ \\
Cinfura (cm) & $109,1 \pm 16,1$ & $81,8 \pm 10,6$ & $<0,001$ \\
VOM - OD $\left(\mathrm{cm}^{3}\right)$ & $13,4 \pm 5,4$ & $13,0 \pm 5,9$ & NS \\
VOM - OE $\left(\mathrm{cm}^{3}\right)$ & $12,6 \pm 6,3$ & $12,7 \pm 5,9$ & NS \\
\hline
\end{tabular}

Variáveis quantitativas com valores expressos em média \pm desvio padrão; IMC: índice de massa corpórea; VOM: volume ovariano médio; OD: ovário direito; OE: ovário esquerdo; Valor $p<0,05$ considerado estatisticamente significativo; NS: não significativo. da avaliação; ciclo menstrual - oligomenorréia (ciclos com intervalos $>35$ dias), amenorréia (ausência do fluxo menstrual por pelo menos 3 ciclos consecutivos), regular (intervalos de $24-32$ dias \pm 3 dias e duração de $3-7$ dias) -; sinais de hiperandrogenismo clínico - acne, alopecia e hirsutismo (definido por índice de Ferriman modificado $\geq 8)^{16}$-; peso $(\mathrm{P})$; altura $(\mathrm{A})$; IMC - definido como $\mathrm{P}(\mathrm{Kg}) / \mathrm{A}\left(\mathrm{m}^{2}\right)$-; medida da circunferência abdominal; pressão arterial sistólica (PAS) e diastólica (PAD).

Após a avaliação clínica, foram coletados $20 \mathrm{~mL}$ de sangue total em tubo sem anticoagulante. No máximo duas horas após a coleta das amostras, o material foi centrifugado a $2500 \mathrm{rpm}$ em temperatura ambiente (média de $22^{\circ} \mathrm{C}$, com variação entre 18 e $24^{\circ} \mathrm{C}$ ) por 10 minutos para separação do soro. A seguir, o soro foi transferido para tubos cônicos de material plástico (BD-Becton Dickinson, Plymouth, Reino Unido) para manter o $\mathrm{pH}$ e submetido ao processamento.

O hormônio folículo estimulante (FSH), o hormônio luteinizante $(\mathrm{LH})$ e a insulina foram avaliados por quimiluminescência com o aparelho DPC Immulite ${ }^{\circledR} 2000$ (Diagnostic Products Corporation, Los Angeles, CA, EUA); a testosterona total, a $17 \mathrm{OH}$-progesterona (17-OH P) e o sulfato de dehidroepiandrostenediona (DHEAS) foram analisados por radioimunoensaio usando o cintilador Tri Carb 2100 TR (Packard ${ }^{\circledR}$ Instrument Company, Illinois, EUA). A glicemia foi analisada pelo método de oxidação usando-se o kit Konelab 60i e aparelho Wiener Lab ${ }^{\circledR}$ (Rosario, Argentina) em dois momentos: jejum e após duas horas da administração de $75 \mathrm{~g}$ de glicose (teste de tolerância oral a glicose 75).

Em relação ao perfil lipídico, o CT, HDL e TG foram dosados pelo método enzimático através do aparelho BT 3000 plus (Wiener lab ${ }^{\circledR}$, Rosario, Argentina); a concentração sérica de LDL foi calculada a partir da fórmula de Friedewald: $\mathrm{LDL}=\mathrm{CT}-(\mathrm{HDL}+\mathrm{TG} / 5)$, uma vez que não havia dosagem de TG superior a $400 \mathrm{mg} / \mathrm{dL}$ nas amostras das pacientes incluídas ${ }^{17}$.

A ultrassonografia pélvica transvaginal considerou a avaliação do volume ovariano e do número/tamanho dos folículos presentes nestes órgãos; para o cálculo do volume ovariano foi utilizada a fórmula do elipsóide prolato (profundidade x largura x comprimento x 0,5$)^{18}$. A comparação entre os grupos foi realizada através da média do volume dos ovários direito e esquerdo de cada paciente; também foi considerada a morfologia ovariana (presença de 12 ou mais folículos em pelo menos um dos ovários) ${ }^{19}$.

Com base nas características clínicas (irregularidade menstrual e hiperandrogenismo), laboratoriais (testosterona total e/ou DHEAS elevados) e ultrassonográficas (volume e/ou morfologia ovariana) foi realizado o diagnóstico de SOP baseado no Consenso de Rotterdam ESHRE/ 
$\mathrm{ASRM}^{15}$, sendo o hiperandrogenismo clínico definido pela presença do hirsutismo, da alopécia androgênica e/ou da acne; o excesso de androgênios séricos foi representado por níveis de testosterona total $\geq 100 \mathrm{ng} / \mathrm{dL}$ ou DHEAS $>300 \mu \mathrm{g} / \mathrm{dL}$.

A MetS foi definida pelo National Cholesterol Education Program (NCEP) ${ }^{9}$. A avaliação da resistência insulínica (RI) foi realizada por dois métodos: (1) bomeostasis model assessment - insulin resistance (HOMA-IR), isto é, HOMA$\mathrm{IR}=[($ glicemia de jejum em $\mathrm{mg} / \mathrm{dL} X 0,05551) \mathrm{X}$ insulina de jejum em $\mu \mathrm{U} / \mathrm{mL}] / 22,5^{20}$; (2) quantitative insulin sensitivity check index (QUICKI) calculado pela seguinte fórmula: QUICKI = 1/[log (insulina jejum) $+\log ($ glicemia jejum)]. A RI foi definida pela alteração de pelo menos um dos seguintes parâmetros: QUICKI $\leq 0,34^{21}$ ou HOMAIR $>2,71^{22}$ A IG foi definida pela presença de níveis de glicemia $2 \mathrm{~h}$ após a ingestão de $75 \mathrm{~g}$ de glicose dentro da seguinte variação: $\geq 140$ e $<200 \mathrm{mg} / \mathrm{dL}$.

\section{Análise estatística}

A verificação da distribuição normal das amostras foi realizada com o teste de Kolmogorov-Smirnov. Para as variáveis com distribuição normal, utilizou-se o teste $t$ de Student não pareado para comparação. $\mathrm{Na}$ ausência desta característica, o teste utilizado foi Mann-Whitney bicaudal. Para as variáveis qualitativas utilizou-se o teste exato de Fisher. Os dados quantitativos foram representados por média e desvio padrão (dp), as variáveis qualitativas foram demonstradas por frequência e o nível de significância adotado foi de $5 \%$. A análise estatística foi realizada com o auxílio do software SAS 9.0 (SAS Institute Inc., North Carolina University, NC, EUA).

\section{Resultados}

Em relação ao perfil hormonal, os níveis de DHEAS e de 17-OH P foram significativamente mais elevados nas mulheres não obesas $(147,9 \pm 76,1 \mathrm{ng} / \mathrm{dL}$ e $109,7 \pm 77,3 \mathrm{ng} /$ $\mathrm{dL}$, respectivamente) do que nas obesas $(120,9 \pm 76,8 \mathrm{ng} /$ $\mathrm{dL}$ e 73,9 $\pm 54,4 \mathrm{ng} / \mathrm{dL}$, respectivamente). Da mesma forma, os níveis de $\mathrm{LH}$ foram maiores nas não obesas do que nas obesas $(9,4 \pm 6,6 \mathrm{mUI} / \mathrm{mL}$ e $5,5 \pm 3,2 \mathrm{mUI} / \mathrm{mL}$, respectivamente; $\mathrm{p}<0,001$ ) (Tabela 2 ).

Entre os parâmetros do lipidograma, comparando-se as pacientes com SOP obesas e não obesas, observaram-se níveis séricos de CT $(189,8 \pm 35,8 \mathrm{mg} / \mathrm{dL}$ e $172,1 \pm 38,4$ $\mathrm{mg} / \mathrm{dL}$, respectivamente; $\mathrm{p}<0,01)$ e de TG $(145,4 \pm 71,1$ $\mathrm{mg} / \mathrm{dL}$ e $99,3 \pm 54 \mathrm{mg} / \mathrm{dL}$, respectivamente; $\mathrm{p}<0,01)$ significativamente mais elevados nas obesas. Os níveis séricos médios de HDL foram inferiores a $50 \mathrm{mg} / \mathrm{dL}$ em ambos os grupos avaliados, sem diferença significativa entre ambos $(45,2 \pm 23 \mathrm{mg} / \mathrm{dL}$ e $49,2 \pm 11,7 \mathrm{mg} / \mathrm{dL}$, respectivamente em obesas e não obesas) (Tabela 2).
Tabela 2. Caracterização hormonal e bioquímica das pacientes obesas e não obesas com síndrome dos ovários policísticos (SOP)

\begin{tabular}{|c|c|c|c|}
\hline \multirow{2}{*}{ Variável } & SOP obesas $n=128$ & SOP não obesas $n=90$ & \multirow{2}{*}{ Valor $p$} \\
\hline & Média dp & Média dp & \\
\hline FSH (mUI/mL) & $5,2 \pm 1,9$ & $5,4 \pm 1,9$ & NS \\
\hline LH (mUI/mL) & $5,5 \pm 3,2$ & $9,4 \pm 6,6$ & $<0,001$ \\
\hline Prolactina (ng/mL) & $10,9 \pm 6,7$ & $12,7 \pm 8,8$ & NS \\
\hline TSH (UI/mL) & $2,1 \pm 1,1$ & $1,9 \pm 1,0$ & NS \\
\hline Testosterona (ng/dL) & $75,5 \pm 34,6$ & $84,9 \pm 30,7$ & NS \\
\hline DHEAS $(\mu \mathrm{g} / \mathrm{dL})$ & $120,9 \pm 76,8$ & $147,9 \pm 76,1$ & 0,02 \\
\hline 17-OH P (ng/dL) & $73,9 \pm 54,4$ & $109,7 \pm 77,3$ & $<0,001$ \\
\hline Colesterol total (mg/ dL) & $189,8 \pm 35,8$ & $172,1 \pm 38,4$ & $<0,01$ \\
\hline LDL colesterol (mg/ dL) & $115,2 \pm 32,6$ & $103,4 \pm 37,5$ & NS \\
\hline HDL colesterol (mg/ dL) & $45,2 \pm 23,0$ & $49,2 \pm 11,7$ & NS \\
\hline Triglicérides (mg/ dL) & $145,4 \pm 71,1$ & $99,3 \pm 54,0$ & $<0,01$ \\
\hline
\end{tabular}

Variáveis quantitativas com valores expressos em média \pm desvio padrão (dp). FSH: hormônio folículo estimulante; LH: hormônio luteinizante; TSH: hormônio tireoestimulante; DHEAS: dehidroepiandrosterona sulfato; 17-OH P: 17-hidroxiprogesterona; LDL: lipoproteína de baixa densidade; HDL: lipoproteína de alta densidade; valores $p<0,05$ considerado estatisticamente significativo; NS: não significativo.

Tabela 3. Comparação da frequência de resistência insulínica, intolerância à glicose, síndrome metabólica e de diabetes mellitus entres pacientes obesas e não obesas com síndrome dos ovários policísticos (SOP)

\begin{tabular}{lccccc}
\hline Variável & $\begin{array}{c}\text { SOP obesus } \\
\mathbf{n}=128(\%)\end{array}$ & \multicolumn{2}{c}{$\begin{array}{c}\text { SOP não obesas } \\
\mathbf{n}=90\end{array}$} & Valor $\mathbf{p}$ \\
\hline Resistência insulínica & 85 & 66,4 & 22 & 24,4 & $<0,01$ \\
Intolerância à glicose & 39 & 30,5 & 11 & 12,2 & 0,03 \\
Síndrome metabólica & 81 & 63,3 & 15 & 16,7 & $<0,01$ \\
Diabetes melliłus & 17 & 13,3 & 4 & 4,4 & NS \\
\hline
\end{tabular}

Resistência insulínica como quantitative insulin sensitivity check índex (QUICKI) $\leq 0,34$; intolerância à glicose - glicemia coletada após $2 \mathrm{~h}$ da ingestão de $75 \mathrm{~g}$ de glicose entre 140 e $199 \mathrm{mg} / \mathrm{dL}$; síndrome metabólica definida pelos critérios diagnósticos do NCEP-ATP III modificado"; valor $p<0,05$ considerado estatisticamente significativo; NS: não significativo.

As frequências de RI (não obesas: $24,7 \%$ vs obesas: $66,7 \%, \mathrm{p}<0,01$ ), IG (não obesas: $12,2 \%$ vs obesas: $29,9 \%, p=0,03$ ) e MetS (não obesas: $16,4 \%$ vs obesas: $63 \%, \mathrm{p}<0,01)$ foram significativamente maiores entre as pacientes obesas do que não obesas (Tabela 3 ).

\section{Discussão}

Evidenciou-se uma elevada prevalência de distúrbios metabólicos (RI, IG e SM) nas mulheres jovens com SOP na amostra estudada. Estes achados são concordantes com os de outros estudos que evidenciaram ser a presença de SOP associada ao elevado risco para o desenvolvimento de comorbidades metabólicas ${ }^{23,24}$. A prevalência destas três alterações metabólicas (RI, IG e SM) foi significativamente maior nas mulheres com SOP obesas do que nas não obesas, demonstrando que a obesidade está associada à piora do perfil metabólico destas mulheres, como evidenciado em estudos de outras populações analisadas ${ }^{23,24}$. A frequência 
de SM foi quatro vezes maior em mulheres com SOP obesas do que nas não obesas (63,3 e 16,7\%, respectivamente). Observou-se também que as prevalências de IG e de RI foram três vezes maiores em mulheres com SOP obesas do que não obesas.

Considerando-se o total de pacientes avaliadas, cerca de $45 \%$ apresentaram SM no momento em que foi realizado o diagnóstico de SOP. Esta frequência é similar àquela demonstrada nos Estados Unidos ( 43 a $46 \%)^{25,26}$ e maior em relação à prevalência encontrada na Itália $(8 \text { a } 16 \%)^{20}$ e na China (2,3 a $12,2 \%)^{27}$, justificando a possível influência da etnia, ambiente e de hábitos alimentares sobre a prevalência das comorbidades metabólicas relacionadas à SOP. Convém ressaltar que estas prevalências populacionais não consideraram a estratificação das mulheres com SOP pelo IMC, o que, como sugerido pelos nossos dados, está fortemente relacionado a modificações significativas na ocorrência de SM nestas mulheres (16,7 e 63\%, respectivamente, em não obesas e obesas). Outros aspectos cruciais passíveis de influenciar variações importantes na prevalência de SM em mulheres com SOP, são os critérios diagnósticos utilizados. No presente estudo, utilizamos os critérios diagnósticos do US National Cholesterol Education Programme Adult Treatment Panel III (NCEP/ATPIII) com a modificação do valor de corte da glicemia de jejum de $110 \mathrm{mg} / \mathrm{dL}$ para $100 \mathrm{mg} / \mathrm{dL}^{9}$. Salientamos que, no total de mulheres avaliadas, $10,4 \%$ apresentaram glicemia de jejum superior a $110 \mathrm{mg} / \mathrm{dL}$ e $22,2 \%$ apresentam glicemia de jejum maior que $100 \mathrm{mg} / \mathrm{dL}$, evidenciando que a modificação de um único critério diagnóstico pode, isoladamente, influenciar de maneira substancial a prevalência deste distúrbio metabólico.

As alterações clínicas e metabólicas da SOP estão relacionadas principalmente ao hiperandrogenismo ${ }^{3,28} \mathrm{e}$ à RI com hiperinsulinemia compensatória ${ }^{4,5}$. Em nossa casuística, o risco metabólico aumentado para SM em mulheres obesas esteve associado a RI e maiores níveis séricos de triglicérides. Já as pacientes não obesas apresentaram maiores níveis séricos de androgênios (hiperandrogenismo laboratorial) e de LH. Estes resultados também foram confirmados por outros autores ${ }^{29,30}$, justificando a possível etiopatogenia multifatorial das comorbidades metabólicas da SOP ${ }^{1}$.

Embora mulheres com SOP possam antecipar para quarta década de vida o desenvolvimento de hipertensão arterial sistêmica, dislipidemia, MetS e $\mathrm{DM}_{2}^{31,32}$, a frequência de $\mathrm{DM}_{2}$ foi semelhante entre os grupos do presente estudo. Entretanto, é possível que mulheres obesas com SOP apresentem maior risco para o desenvolvimento de $\mathrm{DM}_{2}$, uma vez que este grupo apresentou maior prevalência de RI e IG. Ressaltamos que a ausência de diferença na frequência de $\mathrm{DM}_{2}$ entre os grupos estudados possa ser decorrente tanto do fato da casuística estudada ser pequena considerando-se esta variável, como pelo fato de termos avaliamos mulheres na terceira década, fase da vida precoce para detecção do $\mathrm{DM}_{2}$ em pacientes com $\mathrm{SOP}^{1,3,6}$.

Independente do IMC, a frequência de intolerância à glicose foi elevada entre as mulheres com SOP $(12,2 \mathrm{a}$ $29,9 \%)$. Entretanto, esta frequência foi maior em pacientes obesas (duas vezes e meia maior em relação às mulheres magras/sobrepeso). De modo semelhante, outros autores também demonstraram que mulheres obesas com SOP na Espanha apresentavam frequência de intolerância à glicose duas vezes maior do que as mulheres magras ${ }^{8}$. Apesar disto, esta frequência $(7 \text { a } 14 \%)^{8}$ foi menor do que a frequência apresentada no presente estudo, sugerindo a importância de aspectos étnicos e regionais sobre a prevalência das alterações no metabolismo da glicose em mulheres com SOP.

A dislipidemia é um distúrbio comum em mulheres com SOP, mas o foco nestas pacientes tem sido as alterações relacionadas aos componentes da SM (TG e HDL) ${ }^{33}$. De acordo com os resultados do presente estudo, mulheres obesas com SOP apresentaram níveis séricos mais altos de TG do que as não obesas, característica que associada à redução dos níveis de HDL (observada nas pacientes com SOP, independentemente do IMC) demonstra um padrão de dislipidemia associado à RI e à SM, o que poderia caracterizar um maior risco para o desenvolvimento de aterosclerose $e^{9}$. Rocha et al. ${ }^{34}$ também demonstraram que mulheres obesas com SOP apresentam níveis aumentados de TG em relação a pacientes magras. Além disto, estes autores observaram maior redução do HDL sérico entre as mulheres obesas ${ }^{34}$, diferentemente ao demonstrado em nossa casuística. Entretanto, o critério utilizado para definição de SOP foi diferente do empregado no presente estudo, característica que associada à variedade dos hábitos alimentares, poderia justificar os diferentes resultados.

A utilização do HOMA-IR e do Quick para o diagnóstico da RI pode representar uma limitação deste estudo porque o método padrão ouro para este rastreamento é o teste do clamp euglicêmico ${ }^{35}$. Entretanto, o custo financeiro elevado e o tempo prolongado para realização do clamp limitam o seu uso de rotina na prática clínica, principalmente em serviços públicos. Assim, o HOMA-IR pode ser uma alternativa viável para avaliação da $\mathrm{RI}^{36,37}$, inclusive em mulheres com SOP ${ }^{21}$. Utilizando-se estes índices, observou-se uma frequência maior de RI em mulheres com SOP obesas, concordante com dados de vários outros estudos ${ }^{35-36}$.

Em conclusão, mulheres jovens e obesas com SOP apresentam maior prevalência de RI, IG e SM do que as não obesas, apontando um maior risco para o desenvolvimento de comorbidades metabólicas relacionado à obesidade. Todavia, a prevalência dos distúrbios metabólicos é elevada também na pacientes não obesas, sugerindo que 
a presença da síndrome favoreça o desenvolvimento de comorbidades metabólicas independentemente do IMC. Além do hiperandrogenismo, a RI parece apresentar substancial importância no desenvolvimento da SM e dos distúrbios do metabolismo da glicose, principalmente em mulheres obesas com SOP. Estudos de seguimento devem ser realizados para avaliar se mulheres com SOP têm maior risco para o desenvolvimento de doenças cardiovasculares ao longo da vida, no sentido de fundamentar e estabelecer medidas adequadas de prevenção primária e secundária.
1. Melo AS, Vieira CS, Barbieri MA, Rosa-e-Silva AC, Silva AA, Cardoso VC, et al. High prevalence of polycystic ovary syndrome in women born small for gestational age. Hum Reprod. 2010;25(8):2124-31.

2. Norman RJ, Dewailly D, Legro RS, Hickey TE. Polycystic ovary syndrome. Lancet. 2007;370(9588):685-97.

3. Moran L, Teede H. Metabolic features of the reproductive phenotypes of polycystic ovary syndrome. Hum Reprod Update. 2009; 15(4):477-88.

4. Dokras A, Jagasia DH, Maifeld M, Sinkey CA, VanVoorhis BJ, Haynes WG. Obesity and insulin resistance but not hyperandrogenism mediates vascular dysfunction in women with polycystic ovary syndrome. Fertil Steril. 2006;86(6):1702-9.

5. De Zegher F, Ibáñez L. Prenatal growth restraint followed by catchup of weight: a hyperinsulinemic pathway to polycystic ovary syndrome. Fertil Steril. 2006;86 Suppl 1:S4-5.

6. Stovall DW, Bailey AP, Pastore LM. Assessment of insulin resistance and impaired glucose tolerance in lean women with polycystic ovary syndrome. J Womens Health (Larchmt). 201 1;20(1):37-43.

7. Legro RS, Castracane VD, Kauffman RP. Detecting insulin resistance in polycystic ovary syndrome: purposes and piffalls. Obstet Gynecol Surv. 2004;59(2): 141-54.

8. Luque-Ramírez M, Alpañés M, Escobar-Morreale HF. The determinants of insulin sensitivity, $\beta$-cell function, and glucose tolerance are different in patients with polycystic ovary syndrome than in women who do not have hyperandrogenism. Fertil Steril. 2010;94(6):2214-21.

9. Grundy SM, Cleeman JI, Daniels SR, Donato KA, Eckel RH, Franklin BA, et al. Diagnosis and management of the metabolic syndrome: an American Heart Association/National Heart, Lung, and Blood Institute Scientific Statement. Circulation. 2005; 1 12(17):2735-52.

10. de Groot PC, Dekkers OM, Romijn JA, Dieben SW, Helmerhorst FM. PCOS, coronary heart disease, stroke and the influence of obesity: a systematic review and meta-analysis. Hum Reprod Update. $2011 ; 17(4): 495-500$.

11. Wu B, Fukuo K, Suzuki K, Yoshino G, Kazumi T. Relationships of systemic oxidative stress to body fat distribution, adipokines and inflammatory markers in healthy middle-aged women. Endocr J. 2009;56(6):773-82.

12. Vrbikova J, Hainer V. Obesity and polycystic ovary syndrome. Obes Facts. 2009;2(1):26-35.

13. Gambineri A, Vicennati V, Genghini S, Tomassoni F, Pagotto U, Pasquali $R$, et al. Genetic variation in 11 beta-hydroxysteroid dehydrogenase type 1 predicts adrenal hyperandrogenism among lean women with polycystic ovary syndrome. J Clin Endocrinol Metab. 2006;91(6):2295-302.

14. Galluzzo A, Amato MC, Giordano C. Insulin resistance and polycystic ovary syndrome. Nutr Metab Cardiovasc Dis. 2008; 18(7):511-8.
15. The Rotterdam ESHRE/ASRM-Sponsored PCOS Consensus Workshop Group. Revised 2003 consensus on diagnostic criteria and long-term health risks related to polycystic ovary syndrome (PCOS). Hum Reprod. 2004;19(1):41-7.

16. Ferriman D, Gallwey JD. Clinical assessment of body hair growth in women. J Clin Endocrinol Metab. 1961;21:1440-7.

17. Friedewald WT, Levy IR, Fredrickson DS. Estimation of the concentration of low-density lipoprotein cholesterol in plasma, without use of the preparative ultracentrifuge. Clin Chem. 1972;18(6):499-502.

18. Griffin IJ, Cole TJ, Duncan KA, Hollman AS, Donaldson MD. Pelvic ultrasound measurements in normal girls. Acta Paediatr. 1995;84(5):536-43.

19. Balen AH, Laven JS, Tan SL, Dewailly D. Ultrasound assessment of the polycystic ovary: international consensus definitions. Hum Reprod Update. 2003;9(6):505-14.

20. Matthews DR, Hosker JP, Rudenski AS, Naylor BA, Treacher DF, Turner RC. Homeostasis model assessment: insulin resistance and beta-cell function from fasting plasma glucose and insulin concentrations in man. Diabetologia. 1985;28(7):412-9.

21. Carmina E, Lobo RA. Use of fasting blood to assess the prevalence of insulin resistance in women with polycystic ovary syndrome. Fertil Steril. 2004;82(3):661-5.

22. Geloneze B, Repetto EM, Geloneze SR, Tambascia MA, Ermetice $M N$. The threshold value for insulin resistance (HOMA-IR) in an admixtured population IR in the Brazilian Metabolic Syndrome Study. Diabetes Res Clin Pract. 2006;72(2):219-20.

23. Legro RS, Kunselman AR, Dodson WC, Dunaif A. Prevalence and predictors of risk for type 2 diabetes mellitus and impaired glucose tolerance in polycystic ovary syndrome: a prospective, controlled study in 254 affected women. J Clin Endocrinol Metab. 1999;84(1): 165-9.

24. Yilmaz M, Biri A, Bukan N, Karakoç A, Sancak B, Törüner F, et al. Levels of lipoprotein and homocysteine in non-obese and obese patients with polycystic ovary syndrome. Gynecol Endocrinol. 2005;20(5):258-63.

25. Glueck CJ, Papanna R, Wang P, Goldenberg N, Sieve-Smith L. Incidence and treatment of metabolic syndrome in newly referred women with confirmed polycystic ovarian syndrome. Metabolism. 2003;52(7):908-15.

26. Apridonidze T, Essah PA, luorno M, Nestler JE. Prevalence and characteristics of metabolic syndrome in women with polycystic ovary syndrome. J Clin Endocrinol Metab. 2005;90(4):1929-35.

27. Guo M, Chen ZJ, Macklon NS, Shi YH, Westerveld HE, Eijkemans $\mathrm{M}$, et al. Cardiovascular and metabolic characteristics of infertile Chinese women with PCOS diagnosed according to the Rotterdam consensus criteria. Reprod Biomed Online. 2010;21(4):572-80.

28. Fruzzetti F, Perini D, Lazzarini V, Parrini D, Genazzani AR. Adolescent girls with polycystic ovary syndrome showing different phenotypes 
have a different metabolic profile associated with increasing androgen levels. Fertil Steril. 2009;92(2):626-34.

29. Katsikis I, Karkanaki A, Misichronis G, Delkos D, Kandaraki EA, Panidis D. Phenotypic expression, body mass index and insulin resistance in relation to $\mathrm{LH}$ levels in women with polycystic ovary syndrome. Eur J Obstet Gynecol Reprod Biol. $2011 ; 156(2): 181-5$.

30. Silfen ME, Denburg MR, Manibo AM, Lobo RA, Jaffe R, Ferin M, et al. Early endocrine, metabolic, and sonographic characteristics of polycystic ovary syndrome (PCOS): comparison between nonobese and obese adolescents. J Clin Endocrinol Metab. 2003;88(10):4682-8.

31. Elting MW, Korsen TJM, Bezemer PD, Schoemaker J. Prevalence of diabetes mellitus, hypertension and cardiac complaints in a follow-up study of a Dutch PCOS population. Hum Reprod. $2001 ; 16(3): 556-60$.

32. Moran L, Misso ML, Wild RA, Norman RJ. Impaired glucose tolerance, type 2 diabetes and metabolic syndrome in polycystic ovary syndrome: a systematic review and meta-analysis. Hum Reprod Update. 2010;16(4):347-63.

33. Rotterdam ESHRE/ASRM-Sponsored PCOS consensus workshop group. Revised 2003 consensus on diagnostic criteria and longterm health risks related to polycystic ovary syndrome (PCOS). Hum Reprod 2004;19(1):41-7.

34. Rocha MP, Marcondes JA, Barcellos CR, Hayashida SA, Curi DD, da Fonseca $A M$, et al. Dyslipidemia in women with polycystic ovary syndrome: incidence, pattern and predictors. Gynecol Endocrinol. In press 2010.

35. DeFronzo RA, Tobin JD, Andreas R. Glucose clamp technique: a method for quantifying insulin secretion and resistance. Am J Physiol. 1979;237(3):E214-23.

36. Straczkowski M, Stepie A, Kowalska I, Kinalska I. Comparison of simple indices of insulin sensitivity using the euglycemic hyperinsulinemic clamp technique. Med Sci Monit. 2004; 10(8):CR480-4.

37. Radikova Z. Assessment of insulin sensitivity/resistance in epidemiological studies. Endocr Regul. 2003;37(3):189-94. 